\title{
A Client Oriented Scale of Improvement in Tinnitus for Therapy Goal Planning and Assessing Outcomes
}

DOI: $10.3766 /$ jaaa.17119

\author{
Grant D. Searchfield*†
}

\begin{abstract}
Background: There is a need to develop methods to help clinicians work with clients to select and personalize tinnitus therapies. The use of validated measures to determine treatment success is also essential for research and clinical practice. A goal planning method widely used in audiologic rehabilitation is the client oriented scale of improvement (COSI). A modified version of the COSI has been used to identify tinnitus treatment goals and outcomes (client oriented scale of improvement in tinnitus [COSIT]).
\end{abstract}

Purpose: The aims of this study were to identify treatment goals in a clinic sample and ascertain the convergent validity of the COSIT to three widely used standardized questionnaires.

Research Design: A retrospective evaluation of client treatment goals using thematic analysis and correlational analysis of secondary research data comparing the COSIT to tinnitus handicap questionnaire (THQ), tinnitus handicap inventory (THI), and tinnitus functional index (TFI).

Study Sample: One hundred and twenty-two adult patients and research participants attending the University of Auckland Hearing and Tinnitus Clinic.

Results: Specific treatment goals were categorized into 11 themes. The most common treatment goals ( $>10 \%$ of responses) were: (1) Reducing tinnitus' effects on Hearing. (2) Improved wellbeing and being less depressed. (3) Coping with or controlling the tinnitus. (4) Managing the effect of the environment (context) on tinnitus. (5) Improving sleep. (6) Understanding tinnitus. Individuals differed in their complaints and priorities for treatment. The COSIT showed moderate convergent validity with the THQ, THI, and TFI indicating that the total scores measured similar constructs.

Conclusions: The COSIT is a pragmatic method for determining tinnitus treatment goals and priorities in a format that should be familiar to audiologists.

Key Words: client oriented scale of improvement, goal planning, questionnaire, tinnitus, tinnitus functional index, tinnitus handicap inventory, tinnitus handicap questionnaire

Abbreviations: $\mathrm{COSI}=$ client oriented scale of improvement; COSIT = client oriented scale of improvement in tinnitus; SD = standard deviation; TFI = tinnitus functional index; THI = tinnitus handicap inventory; THQ = tinnitus handicap questionnaire

\section{INTRODUCTION}

$\mathrm{T}$ innitus is a common sensory-cognitive disorder with a complex etiology and pathophysiology (Searchfield, 2014). The importance of understanding the whole person in tinnitus management, not just their otological complaint, was recognized many years ago (Fowler, 1948). The importance of a person's motiva- tion, and expectations, for treatment (Hoare et al, 2014) has reemerged recently, just as research exploring the heterogeneity of tinnitus has expanded (Cederroth et al, 2017) and tinnitus models have recognized the individual's environment, culture, and personality as important contributors to tinnitus (Searchfield, 2014). Audiologists usually manage tinnitus through a combination of counseling, hearing aids, and/or sound therapy (Hoare et al,

*Eisdell Moore Centre, Centre for Brain Research and Audiology Section, The University of Auckland, Auckland, New Zealand; †Brain Research New Zealand, Auckland, New Zealand

Corresponding author: Grant D. Searchfield, Audiology Section, University of Auckland, Tamaki Campus, Auckland 1142, New Zealand; Email: g.searchfield@auckland.ac.nz Zealand.

The author's tinnitus research is currently funded from grants awarded by the American Tinnitus Association and the Oticon Foundation of New 
2014). A potential contribution to the stronger evidence for cognitive behavioral therapy over sound-based interventions is greater focus on individual needs (Mckenna and Irwin, 2008; Searchfield et al, 2017). Whereas the concept of client-centered care may be a common stated approach in tinnitus therapy, a recent review suggested that few sound-based treatments address individual needs beyond considering patients hearing loss or tinnitus pitch (Searchfield et al, 2017).

To be effective in client-centered care it is important to recognize and address problems that are relevant to the person (McKenna, 1987). McKenna (1987) describes this process as goal planning. Goal planning recognizes the client's individual needs and often places therapy decisions with them. The value of goal planning and shared decision making is well established in auditory rehabilitation (Goldstein and Stephens, 1981; Dillon et al, 1987; McKenna, 1987), but does not appear overtly in the tinnitus literature. This approach requires clinicians to take into account the real-world needs of individual tinnitus patients and provide customized therapy options. Information provided to clients can include the basis of the treatment, evidence for effectiveness, speed of effects, and costs (Searchfield et al, 2017). Understanding client expectations to provide realistic goals has been shown to be important in hearing aidbased rehabilitation (Dillon et al, 1987) and may be similarly important for tinnitus rehabilitation.

Commonly, tinnitus questionnaires are used to determine the severity or handicap associated with the individual's tinnitus and some have recently been developed to be sensitive to treatment effects (Meikle et al, 2012; Tyler et al, 2014). The content of these questionnaires is usually based on cases studies, surveys, preexisting questionnaires and expert opinion (Meikle et al, 2012). The tinnitus handicap questionnaire (THQ [Kuk et al, 1990]), tinnitus handicap inventory (THI [Newman et al, 1996]), and tinnitus functional index (TFI [Meikle et al, 2012]), are examples of widely used tinnitus questionnaires. The THQ and TFI have undergone assessment of their validity in our clinical population (Searchfield et al, 2007; Chandra et al, 2017). The TFI retained the same structure as the original version developed in a United States population. The THQ was reduced from 27 to 22 items in our population with low factor loading of five questions (2, 8, 12, 25, and 26) (Searchfield et al, 2007).

Another form of questionnaire, less frequently cited in literature, is primarily used as a treatment selection aid, examples include the self-efficacy for tinnitus management questionnaire (Fagelson and Smith, 2016), the sound therapy option profile (Newman et al, 2008), and the tinnitus activities questionnaire (Tyler et al, 2007). The self-efficacy for tinnitus management questionnaire quantifies the patient's confidence in managing tinnitus across five categories: (a) Routine management. (b) Emotion. (c) Thoughts and interactions. (d)
Tinnitus concepts. (e) Use of technology (Fagelson and Smith, 2016). The sound therapy option profile assesses individual motivation, acceptance, expectations, and willingness to use sound therapy (Newman et al, 2008). The tinnitus activities questionnaire determines the areas in which tinnitus creates problems (emotion, sleep, communication, and/or concentration) that can then be used to focus or tailor treatment (Tyler et al, 2007). These assessment and treatment selection questionnaires all use a closed set of questions. No closed-set questionnaire can be expected to cover all potential problems encountered by people with tinnitus. An alternative approach to set questions is to use an open-ended questionnaire in which patients state their own primary problems and goals for treatment. Tyler and Baker (1983) used an open-ended questionnaire in a self-help group in the United Kingdom; they asked:

"Please make a list of the difficulties which you have as a result of your tinnitus. List them in importance, starting with the biggest difficulties" (Tyler and Baker, 1983, p. 150).

Each problem from 97 respondents was cataloged and then categorized. A weighting corresponding to the order of difficulty that the problem created for the individual was calculated. Fifteen of the most common tinnitus difficulties were allocated to four general themes: effects on hearing, effects on general health, effects on lifestyle, and emotional effects (Table 1). Although responses could be placed in categories the range of problems was diverse.

The COSIT (client oriented scale of improvement in tinnitus) is another open-ended format that has been suggested for goal planning and assessment in tinnitus (Searchfield, 2006). The COSIT (Appendix 1) is a modification of the client oriented scale of improvement (COSI) (Dillon et al, 1987) a tool developed to help clinicians plan rehabilitation, based on the individual's communication needs. Closed-set questionnaires may have at least four deficiencies: (a) Patients did not like completing them, especially if the questions were not relevant. (b) Some clinicians did not like scoring them. (c) Some questions could be difficult to interpret (especially by the elderly). (d) The questions often did not focus enough on individual needs (Dillon, 2001). The COSI addressed item relevance by the individual making their own questionnaire identifying and prioritizing the communication situations causing the greatest problems (Dillon et al, 1987). The clinician can work with the patient to ensure that goals are realistic and direct the priorities of the rehabilitation process (Schum, 1999). But the approach also has disadvantages as the situations are not standardized comparisons making interpretation of group effects of treatment more difficult (Saunders et al, 2005). The method does have face validity in understanding an individual's problem, not the problems from a population sample. Based on a correlational analysis the COSI was 
Table 1. Most Common Difficulties Attributed to Tinnitus (Tyler and Baker, 1983) and COSIT Treatment Themes Identified in the Current Study

\begin{tabular}{|c|c|c|c|}
\hline & $\begin{array}{l}\text { Number of } \\
\text { Responses }\end{array}$ & $\begin{array}{c}\text { Percentage of } \\
\text { Responses }\end{array}$ & $\begin{array}{c}\text { Importance } \\
\text { Weighting }\end{array}$ \\
\hline \multicolumn{4}{|l|}{ Tyler and Baker (1983) } \\
\hline \multicolumn{4}{|l|}{ Difficulty } \\
\hline Getting to sleep & 41 & 56.9 & 3.6 \\
\hline Persistence of tinnitus & 35 & 48.6 & 3.6 \\
\hline Understanding speech & 27 & 37.5 & 3.3 \\
\hline Despair, frustration, and depression & 26 & 36.1 & 2.7 \\
\hline Annoyance, irritation, and ability to relax & 25 & 34.7 & 2.6 \\
\hline Concentration and confusion & 24 & 33.3 & 3.1 \\
\hline Dependence on drugs & 17 & 23.6 & 2.5 \\
\hline Pain/headaches & 13 & 18.0 & 3.0 \\
\hline Worse on awakening in morning & 12 & 16.6 & 3.3 \\
\hline Insecurity, fear, and worry & 12 & 16.6 & 2.6 \\
\hline Avoid noisy situations & 11 & 15.3 & 2.6 \\
\hline Withdraw and avoid friends & 10 & 13.8 & 3.0 \\
\hline Giddiness, balance, and fuzzy head & 10 & 13.8 & 3.0 \\
\hline Understand television & 8 & 11.1 & 1.9 \\
\hline Avoid quiet situations & 8 & 11.1 & 3.0 \\
\hline \multicolumn{4}{|l|}{ Current study } \\
\hline \multicolumn{4}{|l|}{ Cosit goal themes } \\
\hline Hear better & 28 & 17.7 & 2.8 \\
\hline Reduce tinnitus effect on wellbeing and depression & 25 & 15.8 & 3.4 \\
\hline Cope with or control tinnitus & 22 & 13.9 & 3.1 \\
\hline Reduce tinnitus effect on context/environment & 22 & 13.9 & 2.6 \\
\hline Improve sleep & 18 & 11.4 & 4.8 \\
\hline Understand tinnitus & 16 & 10.1 & 3.5 \\
\hline Improve quality of life & 7 & 4.4 & 3.6 \\
\hline Improve sound tolerance & 6 & 3.8 & 3.5 \\
\hline Reduce tinnitus effect on cognition/concentration & 6 & 3.8 & 3.0 \\
\hline Reduce or remove annoyance of tinnitus & 4 & 2.5 & 4.8 \\
\hline Change the tinnitus "sound" & 4 & 2.5 & 3.8 \\
\hline
\end{tabular}

Note: The importance weighting is an indication of the severity of the problem (Tyler and Baker, 1983) or prioritization for treatment (COSIT). The weighting functions were calculated in a similar manner but cannot be directly compared across studies, as Tyler and Baker (1983) participants were able to list more than five items.

found to provide similar evidence to the benefit of hearing aids as standardized questionnaires (Dillon et al, 1987).

The COSIT was designed based on the principles and format of the COSI with modifications specific to tinnitus. In the original, COSI clients were asked to nominate up to five situations in which they would like to hear better. The COSIT changed this to "please list five improvements you hope to realize with tinnitus therapy." On the COSI form (Dillon et al, 1987) there was space to indicate two responses to each communication goal: (a) Degree of change (worse, no difference, slightly better, and much better). (b) Final ability with hearing aid person can hear (hardly ever, 10\%; occasionally, 25\%; half the time, $50 \%$; most of the time, $75 \%$; and almost always, 95\%). The COSIT form is modified from the COSI by adding a clarification statement alongside the change score ("with the tinnitus therapy my tinnitus is..." [worse-much better]) and a score has been added (1-5) under each category. The wording of the final score response header also had to change from the COSI to reflect that not experiencing tinnitus was a good result. The statement was changed to: Final result (with therapy) "I am annoyed by my tinnitus..." (almost always, most of the time, half of the time, occasionally, and hardly ever). A score was again added (1-5) under each category.

Searchfield et al (2017) identified a need for a method that helped clinicians and clients work collaboratively to identify treatment goals and then test success in meeting them. Although the COSIT has been used for many years in our clinic (Searchfield, 2006) for this purpose, it had never been formally evaluated. This study investigated the validity of a tinnitus version of Dillon et al (1987) COSI relative to the THQ, THI, and TFI.

\section{METHODS}

$\Upsilon$ his study consists of a retrospective evaluation of 1 four data samples from a total of 122 participants. Sample 1. A convenience sample of 40 participants attending the University of Auckland Hearing and Tinnitus 
clinic who completed the COSIT on their first appointment. Their data were used to characterize common tinnitus treatment goals. Sample 2. Secondary analysis of COSIT data obtained alongside THQ responses, but not included in, a study evaluating tinnitus treatment (Searchfield et al, 2010). Sample 3. Collapsing of COSIT secondary data obtained alongside THI responses from a study investigating multisensory training for tinnitus (Spiegel et al, 2015) and unpublished data exploring the same training with the use of fluoxetine (a selective serotonin reuptake inhibitor). Sample 4. COSIT and TFI responses from the same sample as sample 4 but with the addition of data from a study exploring the effects of frequency compression hearing aids on tinnitus (Hodgson et al, 2015). The COSIT data were not included in the published studies, as the method had not yet been validated. All studies were approved by the University of Auckland Human Participants Ethics Committee.

\section{Participants}

A total of 122 adults participated in this study. Sample 1 consisted of 40 participants 25 males and 15 females with an average age of 63 (standard deviation [SD] = 13)\}. Sample 2 consisted of 33 participants (24 males and nine females with an average age of 63 [SD $=11]$ ). Sample 3 consisted of 35 participants (26 males and nine females with an average age of 57 [SD =10]). Sample 4 consisted of 49 participants ( 36 males and 13 females with an average age of 59 [SD $=9]$ ). The participants in sample 3 were also included in sample 4 .

\section{Procedures}

\section{Audiometry}

Audiometry $(0.25-16 \mathrm{kHz})$ was undertaken using a twochannel audiometer using supraaural (Telephonics, TDH 50P, Framingdale, NY) or insert headphones (E.A.RTONE 3A) $(0.25-8 \mathrm{kHz})$ and high frequency circumaural headphones (Sennheiser HAD200, Germany) (9-16 kHz). Audiometry was performed using the modified HughsonWestlake procedure (Carhart and Jerger, 1959).

\section{THQ}

The THQ is a 27-item self-assessment scale (Kuk et al, 1990) that assesses the handicap caused by tinnitus in typical everyday situations. Participants were instructed to use numbers between 0 and 100 to indicate how much they agreed or disagreed with each item, where 0 corresponded to strongly disagree and 100 to strongly agree. It was scored as an average (between 0 and 100). Responses to 22 questions were used for the final analyses. Five questions (items 2, 8, 12, 25, and 26) were not used as a validation involving New Zealand participants indi- cated significantly lower internal consistency for these items (Searchfield et al, 2007). Item 3 had reversed scoring and was subtracted from 100 before the mean score was calculated.

\section{THI}

The THI (Newman et al, 1996) is a self-report questionnaire comprising 25 questions. The participants were required to answer "yes" (scored as 4), "sometimes" (scored as 2), or "no" (scored as 0 ) to questions designed to identify problems caused by their tinnitus in day-to-day activities. A total score was calculated as the sum of response to each item.

\section{TFI}

The TFI (Meikle et al, 2012) consisted of 25 items and eight subscales. A 0-10 point Likert scale was used to measure the response to each item. The overall TFI can range from 0 to 100 and was calculated by summing the responses obtained from all questions, dividing by the number of questions answered, and multiplying by 10 .

\section{COSIT}

The COSIT is a modified version of the COSI (Dillon et al, 1987). It is an open-ended questionnaire. The researcher asked the participant to nominate up to five goals for tinnitus treatment. They were asked to provide as much detail as possible. Unrealistic goals such as "a cure" were discouraged. Vague goals were clarified with a request for more detail. Once treatment goals were established participants were asked to rate the priority of goals (from 1 up to 5). At posttreatment appointments (samples 2, 3, and 4) participants were asked two questions for each goal: Question 1. With the therapy your tinnitus is...? (response options of worse, no difference, slightly better, better, and much better) this was labeled "degree of change." Question 2. "with tinnitus therapy you are annoyed by your tinnitus...?" (almost always, most of the time, half of the time, occasionally, and hardly ever) this was labeled "final score."

\section{Analysis}

For sample 1 COSIT goals were categorized into themes (stage A, step 2 [Attride-Stirling, 2001]) along with the ranking of the problem. An average importance weighting was calculated for each goal as the product of the number of times participants responses were in a theme, multiplied by the priority of that goal ( 5 for 1st priority to 1 for 5th priority). For example, if the goal was "coping with tinnitus" and the participant rated it as their second goal the weighted score for "coping with tinnitus" would be 4 . 
For samples 2, 3, and 4, two COSIT scores were calculated for each participant; The average degree of change (across goals) and the average final result with therapy (across goals). Total scores were calculated for the THQ, THI, and TFI before and after the intervention. COSIT average change scores were then compared with the average change in the other questionnaires (difference between before and after) and the COSIT final result was compared with the questionnaire scores after intervention. A correlation analysis was undertaken to determine the convergent validity of the COSIT to the other questionnaires using SPSS v.21 (IBM Statistics, Armonk, NY). Past research indicated that the weighing of the COSI improvements by priority did not change its correlation to other measures (Dillon et al, 1987) so it was not applied for correlations in this study. It was hypothesized that: (a) The COSIT final scores and the COSIT changes scores would be moderatelystrongly correlated. (b) The final THQ, THI, and TFI scores would be moderately correlated with the COSIT final score. (c) The difference between pre- and postintervention THQ, THI, and TFI scores with the COSIT would be moderately correlated.

\section{RESULTS}

\section{Treatment Goal Categorization}

Eleven goal themes were identified (Table 1). Most participants identified three goals $(90 \%)$ whereas almost half (42\%) identified five goals. Those identifying more goals tended to be more specific as to what they wanted to achieve from treatment. Although individual statements varied in their specific statements, they were relatively easily categorized, for example:

\footnotetext{
"I want to be able to read and relax when I am in a quiet environment."

"I want to read the newspaper and not be aware of the tinnitus when I am in quiet."
}

These responses were coded as: reduce tinnitus effect on context/environment.

"I want assurance nothing is physically wrong in my brain."

"I want to understand what tinnitus is."

These were coded as: understanding.

There were similarities in the difficulties identified by Tyler and Baker (1983) and the COSIT treatment goals. Tyler and Baker's (1983) top five weighted problems (a combination of times reported and priority) were: (a) Difficulty getting to sleep. (b) Persistence of Tinnitus. (c) Understanding speech. (d) Worse on awakening in morning. (e) Concentration/confusion. The COSIT weighted priorities were: (a) Improve sleep. (b) Reduce annoyance of tinnitus. (c) Change the tinnitus sound. (d) Improve quality of life. (e) (two themes with equal weighting) (i) Understanding tinnitus. (ii) Improved sound tolerance.

\section{COSIT Distribution and Sample Characteristics}

The total COSIT score distribution (Figure 1A) was skewed toward the final problem of tinnitus being minor (50\% of responses were four [I am annoyed by my tinnitus occasionally] or five [I am annoyed by my tinnitus hardly ever] indicating that tinnitus was no longer a large problem). COSIT change scores were skewed toward the treatment having no effect (score of 2). The distribution of COSIT scores for each sample was similar (Figures 1B and C). The samples were similar in age and audiometry, although sample 2 for the analysis of the THQ had slightly poorer low-frequency thresholds (Appendix 2).

\section{Correlation Analysis}

Most hypotheses were confirmed. There was a moderatelow correlation between the COSIT final and COSIT change score $(r=0.418, p<0.001)$. The COSIT final score moderately correlated with the final TFI score $(r=-0.615, p<0.001)$ and final THI score $(r=-0.595$, $p<0.001)$ but not the final THQ score $(r=-0.245$, $p=0.18$ ). The COSIT change score moderately correlated with the change in THQ $(r=0.525, p<0.005)$ and TFI $(r=0.498, p<0.001)$ with a moderate-low correlation with the THI $(r=0.351, p<0.05)$.

\section{DISCUSSION}

$\mathrm{T}$ he COSIT was found to have moderately convergent validity with several established closed-set questionnaires. The treatment goals identified were consistent with a previous study exploring difficulties encountered by persons with tinnitus (Tyler and Baker, 1983). The samples shared similar problems. Although Tyler and Baker (1983) study of a tinnitus self-help group in the United Kingdom used an open response format, the question asked did slightly differ from that asked for the COSIT. Tyler and Baker (1983) were interested in problems whereas the question asked in this study concerned treatment goals. Although both studies had a similar age demographic there were differences in gender, decade of social-cultural history $(\sim 30$ years difference when the studies were undertaken), and country of sample (United Kingdom versus New Zealand). Different goals are likely to be identified in different demographics and cultures. Because the COSIT does not use a priori questions, it may be less susceptible to translation or culture specific bias than 

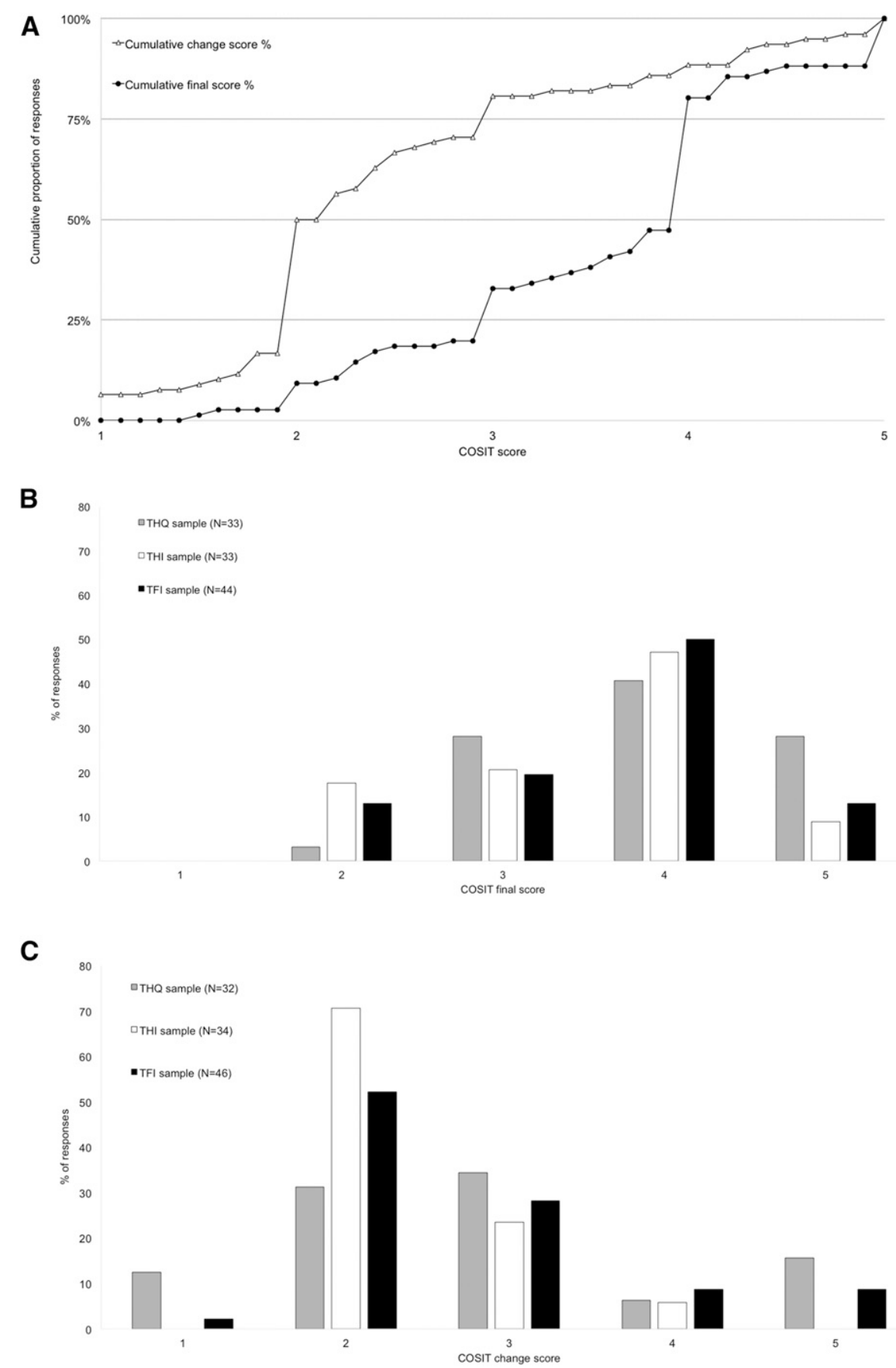

Figure 1. (A) Cumulative frequency distribution of COSIT scores across samples 2, 3, and 4. COSIT final scores and change scores are shown. The COSIT final scores are skewed toward tinnitus not being a problem after intervention, whereas the COSIT change scores indicate that many participants did not feel the tinnitus changes with treatment. The categorical nature of responses across in a question containing five or fewer items has resulted in the average responses clustering at the category score. (B) Frequency distribution of COSIT final responses and (C) COSIT change scores (\%) for each study sample.

other questionnaire formats. It, however, may be vulnerable to interviewer bias and between-clinician differences. In these respects, principles of quality and validity used in qualitative research should be examined (Golafshani, 2003).

Tyler and Baker (1983) identified that sleep and persistence of tinnitus had the greatest importance weighting amongst the 15 most common problems. This was similar for the COSIT treatment goals; sleep and annoyance received the greatest importance weighting. Whereas many of the common problems and COSIT treatment goals were similar, one common problem in Tyler and Baker (1983) sample was absent from our data. Pain/headaches was a frequently reported problem, yet assistance with pain or headaches was not identified as a treatment goal. A search of raw text responses on the COSIT did not identify 
"pain" or "headaches" in any statement. It is possible that the participants, or clinician, viewed pain as independent of tinnitus and so was not reported. If pain was related to sound intolerance it is possible it was captured in that theme. Comparisons across qualitative measures are difficult because of potential differences in the perspectives and what we bring to the study as researchers (Golafshani, 2003). The coding and collapsing into themes may place participants' statements containing several items into one or other theme based on unintentional bias from the researcher. For example, the example statement "I want to read the newspaper and not be aware of the tinnitus when I am in quiet" was coded as reduce tinnitus effect on context/environment. It could have also been coded as reduce tinnitus effect on cognition/concentration. This would have required interpretation that the tinnitus was affecting concentration, which is not explicitly stated. Because the underpinning philosophy can make comparisons of qualitative data difficult (Golafshani, 2003) the comparisons in Tyler and Baker's (1983) results and the COSIT results should be taken at simple face value, that is, clients vary in their difficulties and treatment goals. Some problems are more common, but are viewed as less of a priority for treatment by the individual. The open-ended format does have the disadvantage of relying on patients recall. Questionnaires using a priori questions may prompt individuals to consider how a particular feeling or situation affects their tinnitus, that otherwise they may have forgotten (Tyler and Baker, 1983). A counter perspective is that questions asked may give rise to negative thoughts that the individual had never considered. In our practice we have not adopted the Tinnitus Reaction Questionnaire (Wilson et al, 1991) for general use because of the negative response of clients about several of its items, in particular, its request for a response to the statement "My tinnitus has led me to think about suicide."

The most common problems need not be the highest priority for treatment. Tyler and Baker (1983) found that "tinnitus on awakening" was ranked 3rd equal based on weighting for priority but was the 9th most common problem. In the current study "reduce or remove the annoyance of tinnitus" was 1st equal weighted priority but was the least frequent treatment goal. Hearing better was a common goal, but it received a lower priority than most treatment goals. Although interesting to consider group effects, the real strength of openended questionnaires such as the COSIT is at the individual level.

\section{COSIT Distribution}

The COSIT change score and COSIT final score had different cumulative frequency distributions. Whereas many participants reported little or no benefit from intervention, similar numbers reported their tinnitus treatment goal was no longer a problem. The reasons for this divergence were not explored when data were acquired, but there are several plausible explanations. It is possible that the treatment had no effect and the final rating simply reflected that the tinnitus was not a large problem. This would be unusual because the participant had listed each item as a problem. Another explanation is that the final tinnitus was better, but the participant did not believe the treatment was responsible. No one reported a score of 1 (tinnitus worse) to the final rating COSIT question. There were no obvious differences in COSIT score distribution in the three samples for either change or final score. The difference between change and final scores was greater than hypothesized with a significant, but moderate-weak, correlation between the scales; a strong correlation was hypothesized.

\section{Convergent Validity}

The COSIT final score was most strongly correlated with the TFI and least, and statistically not significantly, with the THQ score. The result suggests that the COSIT final score was measuring a similar construct to the TFI and THI, but the COSIT final and the THQ score after treatment measured different aspects of tinnitus. This was reversed in the case of the COSIT change score, it was most strongly correlated to the difference between pre- and post-intervention THQ scores. The COSIT change score was also significantly correlated with the changes in the TFI and THI. The moderate correlations are indicative of similarities in the measures, but they are not measuring exactly the same aspects of tinnitus. In research, the use of the COSIT along with a standard questionnaire would be useful to ensure coverage of important domains of tinnitus.

\section{Application}

This study did not examine if the COSIT provided benefit in the process of goal planning. It is important that the benefits and shortcomings of the COSIT in selecting treatments be examined. The following description of clinical use of the COSIT is to enable clinicians and researchers to use the tool. The individual describes the problems with the clinician framing the goal in a positive light while discouraging unrealistic goals. An outcome of setting goals is the establishment of a therapy plan suited to the participant's priorities. The intent is that the plan should address each goal, and need not follow a single specific treatment paradigm for all goals, rather each goal is considered external referral or internal if in a multidisciplinary environment (McKenna, 1987). Reflection on the problems attributed to tinnitus could be therapeutic and help to clarify the problems that individuals face, becoming a focus for counseling (Tyler and Baker, 1983). We believe those factors of the individual's complaint likely to be driving other symptoms should 
be addressed first. A good clinician will also recognize that addressing lower priority goals may affect higher priorities. For example, improving hearing may be a lower rated priority in the COSIT, but hearing aids might assist cognition or annoyance goals (Searchfield et al, 2017). It is possible for goals to be managed sequentially or in parallel. Priority may need to be given to a particular goal that may limit the effectiveness of any planned therapy for another goal. An example of this may be a circumstance where an individual is experiencing distressing anxiety. In this case addressing hearing or the annoyance of tinnitus may have to wait until mental health difficulties are addressed. The selection of treatments to use immediately and as or if tinnitus changes may also be empowering to the patient. In clinical practice determination of individual needs and priorities, alongside assessment measures such as pure-tone audiometry, pitch matching, and standard questionnaires may reduce the risk for ineffective treatment. If effective, the COSIT may also reduce the time required for treatment reducing stress, anxiety, and loss of hope for the sufferer. These thoughts need to be systematically evaluated in a study examining sound therapy and rehabilitation for tinnitus (Searchfield et al, 2017).

This study was undertaken retrospectively and consisted of an evaluation of the COSIT with other questionnaires independent of each other, that is, the groups comparing COSIT and THQ, COSIT and TFI, and COSIT and THI were not the same; a prospective study using repeated measures in the same population is needed. Although the COSIT uses a quantitative assessment scale, the determination of goals shares some characteristics with qualitative methods. The testretest reliability and inter-examiner reliability of the COSIT should be examined from a qualitative view (Golafshani, 2003). Because of its mix of models, individual item comparisons of the COSIT with subscales of the other questionnaires is difficult and possibly misleading. We chose not attempt such a comparison in this study.

\section{CONCLUSIONS}

A s a preliminary, and retrospective, examination of the COSIT, this study has covered the basic components of questionnaire validity. Treatment goals were consistent with tinnitus problems identified in a previous study (Tyler and Baker, 1983). The scores show sufficient convergent validity with existing standardized questionnaires for the COSIT to be used as an outcome measure; however it does need to be tested further.

Acknowledgments. The author would like to thank Chris Jerram and Alexia Searchfield for the COSIT text transcription and data entry.

\section{REFERENCES}

Attride-Stirling J. (2001) Thematic networks: an analytic tool for qualitative research. Qual Res 1:385-405.

Carhart R, Jerger JF. (1959) Preferred method for clinical determination of pure-tone thresholds. J Speech Hear Disord 24: $330-345$

Cederroth CR, Kähler AK, Sullivan PF, Lopez-Escamez JA. (2017) Genetics of tinnitus: time to biobank phantom sounds. Front Genet 8:110.

Chandra N, Chang K, Lee A, Shekhawat GS, Searchfield GD. (2017) Psychometric validity, reliability, and responsiveness of the tinnitus functional index. J Am Acad Audiol, in press.

Dillon H. (2001) Hearing Aids. Sydney: Boomerang Press.

Dillon H, James A, Ginis J. (1987) Client oriented scale of improvement (COSI) and its relationship to several other measures of benefit and satisfaction provided by hearing aids. J Am Acad Audiol 8:27-43.

Fagelson MA, Smith SL. (2016) Tinnitus self-efficacy and other tinnitus self-report variables in patients with and without posttraumatic stress disorder. Ear Hear 37:541-546.

Fowler EP. (1948) The emotional factor in tinnitus aurium. Laryngoscope 58:145-154.

Golafshani N. (2003) Understanding reliability and validity in qualitative research. Qual Rep 8:597-606.

Goldstein D, Stephens S. (1981) Audiological rehabilitation: management model I. Audiology 20:432-452.

Hoare DJ, Searchfield GD, El Refaie A, Henry JA. (2014) Sound therapy for tinnitus management: practicable options. J Am Acad Audiol 25:62-75.

Hodgson S-A, Herdering R, Singh Shekhawat G, Searchfield GD. (2015) A crossover trial comparing wide dynamic range compression and frequency compression in hearing aids for tinnitus therapy. Disabil Rehabil Assist Technol 12(1):97-103.

Kuk FK, Tyler RS, Russell D, Jordan H. (1990) The psychometric properties of a tinnitus handicap questionnaire. Ear Hear 11: $434-445$.

McKenna L. (1987) Goal planning in audiological rehabilitation. Br J Audiol 21:5-11.

Mckenna L, Irwin R. (2008) Sound therapy for tinnitus: sacred cow or idol worship?: an investigation of the evidence. Audiol Med 6:16-24.

Meikle MB, et al. (2012) The tinnitus functional index: development of a new clinical measure for chronic, intrusive tinnitus. Ear Hear 32:153-176.

Newman CW, Jacobson GP, Spitzer JB. (1996) Development of the tinnitus handicap inventory. Arch Otolaryngol Head Neck Surg 122:143-148.

Newman CW, Sandridge SA, Meit SS, Cherian N. (2008) Strategies for managing patients with tinnitus: a clinical pathway model. Semin Hear 29:300-309.

Saunders GH, Chisolm TH, Abrams HB. (2005) Measuring hearing aid outcomes-not as easy as it seems. J Rehabil Res Dev 42: $157-168$

Schum DJ. (1999) Perceived hearing aid benefit in relation to perceived needs. J Am Acad Audiol 10:40-45. 
Searchfield GD. (2006) Hearing aids and tinnitus. In: Tyler R, ed. Tinnitus Protocols. New York, NY: Thieme.

Searchfield GD. (2014) Tinnitus what and where: an ecological framework. Front Neurol 5:271.

Searchfield GD, Durai M, Linford T. (2017) A state-of-the-art review: personalization of tinnitus sound therapy. Front Psychol 8: 1599 .

Searchfield GD, Jerram C, Wise K, Raymond S. (2007) The impact of hearing loss on tinnitus severity. Aust $N Z$ J Audiol 29: 67-76.

Searchfield GD, Kaur M, Martin WH. (2010) Hearing aids as an adjunct to counseling: tinnitus patients who choose amplification do better than those that don't. Int $J$ Audiol 49:574-579.
Spiegel DP, Linford T, Thompson B, Petoe MA, Kobayashi K, Stinear CM, Searchfield GD. (2015) Multisensory attention training for treatment of tinnitus. Sci Rep 5:10802.

Tyler R, Ji H, Perreau A, Witt S, Noble W, Coelho C. (2014) Development and validation of the tinnitus primary function questionnaire. Am J Audiol 23:260-272.

Tyler RS, Baker LJ. (1983) Difficulties experienced by tinnitus sufferers. J Speech Hear Disord 48:150-154.

Tyler RS, Gogel SA, Gehringer AK. (2007) Tinnitus activities treatment. Progr Brain Res 166:425-434.

Wilson PH, Henry J, Bowen M, Haralambous G. (1991) Tinnitus reaction questionnaire: psychometric properties of a measure of distress associated with tinnitus. J Speech Hear Res 34:197-201. 


\section{APPENDIX 1}

\section{Client Oriented Scale of Improvement in Tinnitus (COSIT)}

The COSIT is a tool to assist the audiologist in measuring the success of your tinnitus therapy. Please list five improvements you hope to realize with this therapy. Please be as specific as possible.

Audiologist:

Dates: 1. Needs established

2. Outcome measured

\begin{tabular}{|c|c|c|c|c|c|c|c|c|c|c|c|}
\hline \multirow{3}{*}{\multicolumn{2}{|c|}{$\begin{array}{cc}\text { Indicate } \\
\text { Order of } \\
\text { Specific needs } & \text { Significance }\end{array}$}} & \multicolumn{5}{|c|}{ Degree of Change } & \multicolumn{5}{|c|}{ Final Result (with Therapy) } \\
\hline & & \multicolumn{5}{|c|}{ "With the tinnitus therapy, my tinnitus is ..." } & \multicolumn{5}{|c|}{ "I am annoyed by my tinnitus ..." } \\
\hline & & $\begin{array}{c}\text { Worse } \\
1\end{array}$ & $\begin{array}{c}\text { No } \\
\text { Different } \\
2\end{array}$ & $\begin{array}{l}\text { Slightly } \\
\text { Better } \\
3\end{array}$ & $\begin{array}{c}\text { Better } \\
4\end{array}$ & $\begin{array}{c}\text { Much } \\
\text { Better } \\
5\end{array}$ & $\begin{array}{c}\text { Almost } \\
\text { Always } \\
1\end{array}$ & $\begin{array}{c}\text { Most of } \\
\text { the Time } \\
2\end{array}$ & $\begin{array}{c}\text { Half } \\
\text { the Time } \\
3\end{array}$ & $\begin{array}{c}\text { Occasionally } \\
4\end{array}$ & $\begin{array}{l}\text { Hardly } \\
\text { Ever } \\
5\end{array}$ \\
\hline
\end{tabular}

This questionnaire is a modified form of the COSIT (Dillon et al, 1987, p. 43). Readers are welcome to copy and utilize for their personal use; distributing copies is not permitted without prior approval. 


\section{APPENDIX 2}

Average pure-tone air conduction thresholds for participants ( \pm 1 SEM) in: (A) Sample 2 (THQ) 250-8000 Hz. (B) Sample 3 (THI) 250-16000 Hz and (C) Sample 4 (TFI) 250-16000 Hz. Thresholds at 14000 and $16000 \mathrm{~Hz}$ were beyond the limits of the audiometer intensity range.

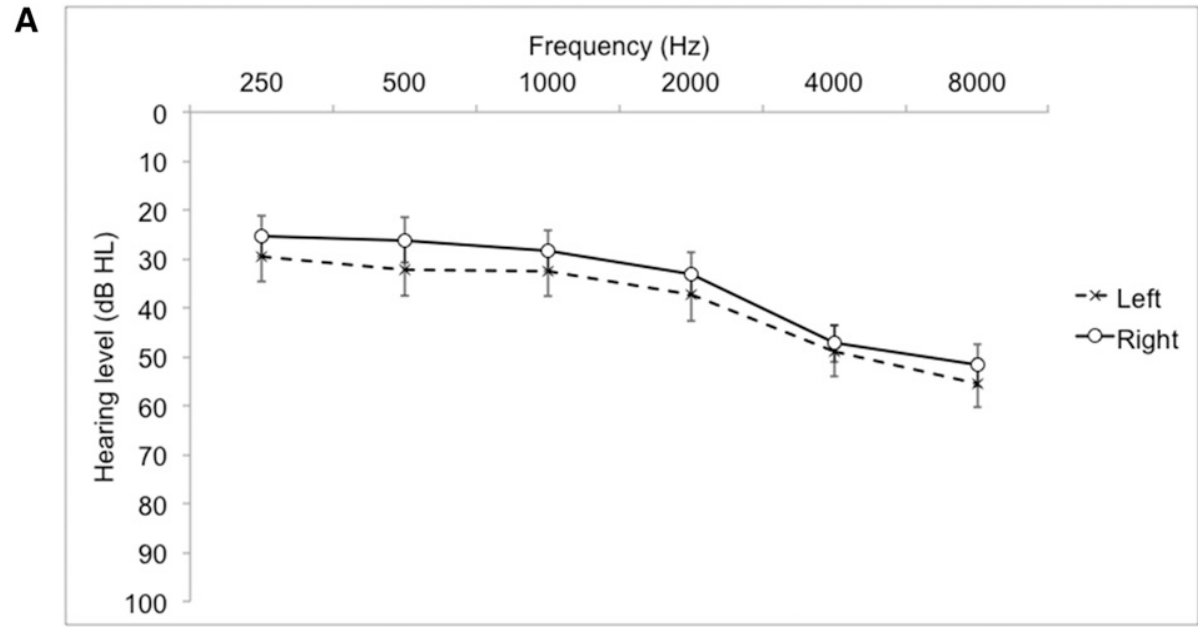

B
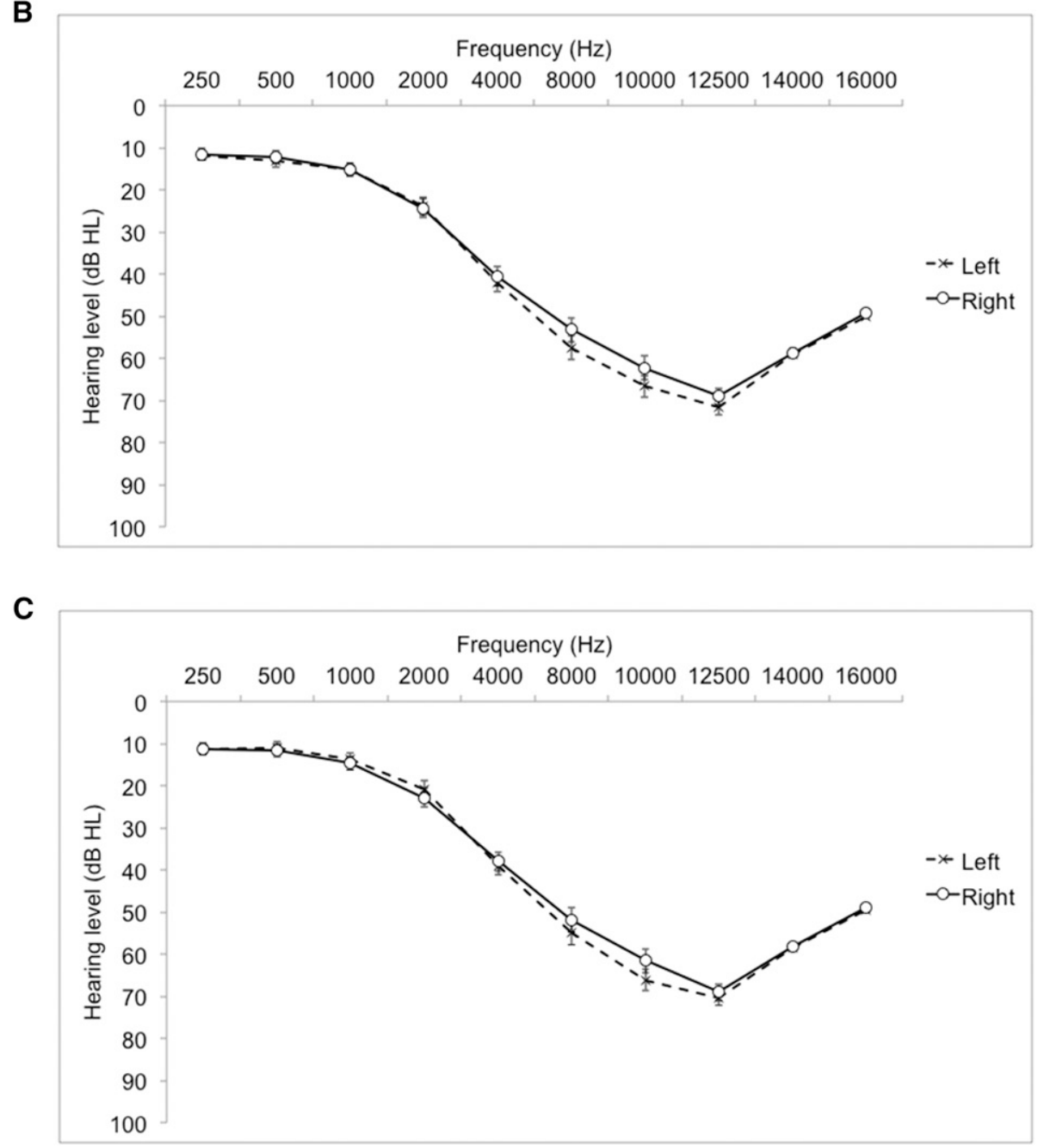